\title{
A role for ITPA variants in the clinical course of pulmonary Langerhans' cell histiocytosis?
}

\section{To the Editors:}

Interstitial lung diseases (ILD) comprise a number of clinical conditions, including sarcoidosis, pulmonary fibrosis and pulmonary Langerhans' cell histiocytosis (PLCH; histiocytosis $\mathrm{X})$. The diagnostic workup for the classification of ILD is often complicated and tedious. PLCH is characterised by the proliferation of Langerhans' cells and their infiltration into pulmonary tissues. In disseminated $\mathrm{LCH}$, additional tissues are affected. PLCH is strongly associated with smoking and the clinical outcome depends upon cessation of smoking [1].

Treatment of ILD often requires corticosteroids and immunosuppressant drugs, including thiopurines and biologicals. It is mandatory to exclude genetic polymorphisms in genes involved in metabolism of thiopurine metabolism, because of the pharmacogenetic consequences of the use of thiopurines. The prospective measuring of erythrocyte enzyme activity of thiopurine $S$-methyltransferase (TPMT) and inosine triphosphatase (ITPase) or molecular analyses of the genes encoding these enzymes is recommended in clinical practice [2].

Aberrant enzyme activity of either TPMT or ITPase gives rise to a broad spectrum of adverse drug reactions (ADR) associated with thiopurines, ranging from therapeutic failure to life-threatening leukopenia [3, 4].

In order to avoid these ADR, we have incorporated pre-treatment screening of TPMT and ITPase of patients with ILD who are candidates for treatment with thiopurines in our hospital.

An unexpectedly high number of decreased ITPase activities in patients with PLCH was found, all of which had an unfavourable outcome. We hypothesised about the role of the ITPA gene in relation to immunity, which might play a key role in this phenomenon.

We examined a total of 105 patients with ILD, referred to the ILD Care Team of the Maastricht University Medical Centre, a tertiary referral centre in Maastricht, The Netherlands for ILD (table 1).

According to the local protocol for the workup of patients with ILD where thiopurine medication is considered, TPMT and ITPase activities in erythrocytes were measured prior to the start of the treatment. When a lowered activity of TPMT or ITPase was detected, molecular analysis of the TPMT and ITPA genes were performed. Reference values for ITPase and ITPA polymorphism distribution were established in a group of 100 anonymous patients from a general hospital population.

TPMT and ITPase activities were measured in erythrocyte lysates, as described previously [5]. Molecular analyses of TPMT and ITPA genes were performed using previously published protocols [6].

The results of the TMPT and ITPase phenotyping and genotyping in the ILD population are displayed in table 1.
ITPase activity was decreased in 50\% (three out of six) PLCH patients. The patients were genotyped and in patients with a decreased ITPase activity this was confirmed by the finding of polymorphic ITPA genotypes, either heterozygous or homozygous (table 2). The distribution of ITPase activity and ITPA polymorphisms in the reference population were consistent with earlier published results [6]. Erythrocyte TPMT activity was normal in the PLCH patients, as confirmed by molecular analysis, revealing the wild type ${ }^{*} 1 /{ }^{*} 1$ genotypes in all patients.

Although the number of patients with $\mathrm{PLCH}$ in our cohort is limited, we detected an unusually high and intriguing prevalence of low erythrocyte ITPase activities and a concomitant higher number of ITPA polymorphisms, i.e. 50\%, compared to the reference population at $11 \%$.

It is unclear whether there is a connection between the absolute ITPase activity and the occurrence of the disease. Earlier reports referring to patients with a complete ITPase deficiency reported no clinical abnormalities, classifying it as a benign condition [7]. Interestingly, the PLCH cases carrying an ITPA polymorphism all demonstrated an unfavourable outcome despite the fact that they all stopped smoking. To date, smoking is associated with the clinical course of PLCH. In general, the clinical features improve after the patient stops smoking. However, our three patients carrying an ITPA polymorphism all deteriorated and no clinical improvement occurred compared to the PLCH patients with the normal genotype. This finding is also clinically relevant regarding the therapeutic options, as patients who do not improve after they quit smoking are sometimes candidates for azathioprine. Therefore, we strongly recommend the assessment of erythrocyte ITPase activity, in addition to TPMT activity, prior to initiating treatment with azathioprine in patients with PLCH.

To the best of our knowledge inosine triphosphate (ITP) does not accumulate in patients with a partial ITPase deficiency. Therefore, clinical pathology cannot merely be explained by accumulation of ITP in carriers of ITPA polymorphisms, but is more likely to be associated with housekeeping or moonlighting functions of the gene or its protein(s).

The role of ITPase in mammalian metabolism is still poorly understood [8]. Its primary role is the pyrophosphohydrolysis of ITP (and deoxy-ITP) to maintain the balance between ITP and inosine monophosphate (IMP), IMP being the key metabolite in the purine interconversion pathway. Tissue distribution of ITPase varies widely, the highest activity is present in tissues with an exocrine function [9].

The other, less highlighted, function of ITPA is its role as a housekeeping gene. ITPase is involved in the maintenance of the degradation of noncanonical purine nucleotide triphosphates, hereby preventing the incorporation of these metabolites into RNA and DNA. Under abnormal physiological circumstances, such as metabolic stress or an inflammatory 
TABLE 1 Distribution of ITPA polymorphisms in interstitial lung diseases (ILD)

\begin{tabular}{lccc} 
& All patients & Lowered ITPase activity & ITPA polymorphisms \\
\hline Pulmonary sarcoidosis (chest radiograph stage IV) & 41 & 8 & $8(19.5)$ \\
IPF & 50 & 3 & $3(6.0)$ \\
PLCH & 6 & 3 & $3(50)$ \\
Other causes of ILD & 100 & 4 & $4(50)$ \\
Reference population $^{\#}$ & 8 & 11 & $11(11)$ \\
\hline
\end{tabular}

Data are presented as $\mathrm{n}$ or $\mathrm{n}(\%)$. ITPase: inosine triphosphatase; IPF: idiopathic pulmonary fibrosis; PLCH: pulmonary Langerhans' cell histiocytosis. \#: definition of reference population presented in the main text. ": the four cases with an ITPA polymorphism were suffering from drug-induced pneumonitis ( $n=2)$ due to azathioprine, chronic extrinsic allergic alveolitis $(n=1)$, and lymphangioleiomyomatosis $(n=1)$.

response, proper functioning of housekeeping genes is pivotal for cellular maintenance. In patients carrying one or more polymorphic ITPA alleles, it can be speculated that the housekeeping function may be compromised and inflammatory responses cannot be countered properly, resulting in enhanced disease activity. In PLCH there is excessive eosinophilic granuloma formation, thereby corrupting the immune response. Although little is known about ITPase activity in progenitor cells of eosinophils and macrophages, it can be speculated that a wild type ITPA genotype is crucial to maintain adequate housekeeping in these cells. Comparing ITPase deficiency with two other inherited disorders in purine metabolism, adenosine deaminase and purine nucleoside phosphorylase deficiency, makes this line of thought even more compelling. Both conditions cause severe immune disease, resulting in severe combined immune deficiency and T-cell immunodeficiency respectively. The concept of a potential role of ITPA in immunity is further strengthened by the fact that two proteins encoded by ITPA were recognized by cytotoxic T-cells. Whether or not these immunogenic proteins are the products of alternative splicing remains unclear. However, as was shown by ARENAS et al. [10], alternative mRNA splice variants are present in significantly higher amounts in patients carrying the ITPA $94 \mathrm{C}>\mathrm{A}$ polymorphism than in wild type individuals. A direct link between ITPase and smoking seems highly unlikely. One may argue, however, that toxic compounds originating from (tobacco) smoke cause oxidative stress in exposed tissues, generating noncanonical nucleotides. ITPase is required to scavenge these toxic compounds. Expression of ITPase in lung tissue is low, which may imply that under metabolic stress the presence of ITPA polymorphisms results in insufficient ITPase activity thus compromising the cellular capability to neutralise toxic, noncanonical, nucleotides.

In conclusion, we hypothesise that ITPA polymorphisms are associated with an unfavourable clinical outcome in PLCH. Future investigations are required to confirm the high prevalence of ITPA polymorphisms in PLCH patients. Moreover, the prognostic value should be evaluated in a larger cohort, focussing especially on the association with smoking. Furthermore, the potential role of ITPase in inflammatory modulating cells needs to be elucidated. These investigations may give more insight in the real physiological role of ITPase and the clinical effect of polymorphisms in the ITPA gene.

\section{J.A. Bakker*,\#,+, J. Bierau ${ }^{\#,+}$, M. Drent ${ }^{\#, \oplus,+}$}

*Laboratory for Biochemical Genetics, Dept Clinical Genetics, ${ }^{*}$ ILD Care Team, and ${ }^{\circledR}$ Dept of Respiratory Medicine, Maastricht

TABLE 2 Inosine triphosphatase (ITPase) activity, ITPA genotype and clinical outcome in pulmonary Langerhans' cell histiocytosis (PLCH) patients

\begin{tabular}{|c|c|c|c|c|c|c|}
\hline Sex & $\begin{array}{c}\text { Age at diagnosis } \\
\text { yrs }\end{array}$ & $\begin{array}{l}\text { Follow-up } \\
\text { months }\end{array}$ & Smoking status & $\begin{array}{c}\text { ITPase activity }{ }^{\#} \\
\mu \mathrm{mol} \cdot \mathrm{mmol}^{-1} \mathrm{Hb} \cdot \mathrm{h}^{-1}\end{array}$ & ITPA genotype & Clinical outcome \\
\hline $\mathrm{F}$ & 17 & 70 & Stopped & 3.15 & Wt & Favourable ; clinically improved \\
\hline M & 38 & 19 & Stopped & 4.60 & Wt & Favourable ; clinically improved \\
\hline M & 52 & 21 & Smoking & 2.89 & Wt & Unchanged \\
\hline $\mathrm{F}$ & 32 & 27 & Stopped & 0.03 & c.94AA & Unfavourable ${ }^{+}$; awaiting lung transplantation \\
\hline M & 36 & 49 & Stopped & 0.79 & c.94CA & Unfavourable $^{+}$ \\
\hline M & 54 & 37 & Stopped & 0.28 & $\begin{array}{c}\text { c. } 94 \mathrm{CA} / \\
\text { g.IVS2+21AC }\end{array}$ & Unfavourable $^{+}$ \\
\hline
\end{tabular}

\footnotetext{
\# : reference values: $4.0-10.0 \mu \mathrm{mol} \cdot \mathrm{mmol}^{-1} \mathrm{Hb} \cdot \mathrm{h}^{-1}(\mathrm{n}=100) ;{ }^{\bullet}$ : favourable is defined as substantial improvement of the high resolution computed tomography $(\mathrm{HRCT})$ and $>10 \%$ improvement of the diffusing capacity of the lung for carbon monoxide $(D L, C O){ }^{+}$: unfavourable is defined as a worse evaluation of the $\mathrm{HRCT}$, a $>10 \%$ decrease of the $\mathrm{DL}, \mathrm{CO}$ and desaturation during a 6-min walking test.
} 
University Medical Centre, Maastricht, The Netherlands

${ }^{+}$These authors contributed equally.

Correspondence: J.A. Bakker, Laboratory for Biochemical Genetics, Dept Clinical Genetics, Maastricht University Medical Centre, P. Debyelaan 25, 6229 HX Maastricht, The Netherlands. E-mail: jaap.bakker@mumc.nl

Statement of Interest: None declared

\section{REFERENCES}

1 Harari S, Caminati A. Pulmonary Langerhans' cell histiocytosis. Eur Respir Mon 2009; 46: 155-175.

2 Weinshilboum R, Wang L. Pharmacogenomics: bench to bedside. Nat Rev Drug Discov 2004; 3: 739-748.

3 Bakker JA, Bierau J, Drent M. Therapeutic regimens in interstitial lung disease guided by genetic screening: fact or fiction? Eur Respir J 2007; 30: 821-822.
4 Coulthard S, Hogarth L. The thiopurines: an update. Invest New Drugs 2005; 23: 523-532.

5 Bierau J, Bakker JA, Lindhout M, et al. Determination of ITPase activity in erythrocyte lysates obtained for determination of TPMT activity. Nucleosides Nucleotides Nucleic Acids 2006; 25: 1129-1132.

6 Sumi S, Marinaki AM, Arenas M, et al. Genetic basis of inosine triphosphate pyrophosphohydrolase deficiency. Hum Genet 2002; 111: 360-367.

7 Vanderheiden BS. Human erythrocyte "ITPase": an ITP pyrophosphohydrolase. Biochim Biophys Acta 1970; 215: 555-558.

8 Bierau J, Lindhout M, Bakker JA. Pharmacogenetic significance of inosine triphosphatase. Pharmacogenomics 2007; 8: 1221-1228.

9 Lin S, McLennan AG, Ying K, et al. Cloning, expression, and characterization of a human inosine triphosphate pyrophosphatase encoded by the ITPA gene. J Biol Chem 2001; 276: 18695-18701.

10 Arenas M, Duley J, Sumi S, et al. The ITPA c.94C $>$ A and g.IVS2 $+21 \mathrm{~A}>\mathrm{C}$ sequence variants contribute to missplicing of the ITPA gene. Biochim Biophys Acta 2007; 1772: 96-102.

DOI: $10.1183 / 09031936.00026610$

\section{Quadriceps myostatin expression in COPD}

\section{To the Editors:}

Quadriceps muscle dysfunction is well recognised in chronic obstructive pulmonary disease (COPD), and is associated with impaired exercise capacity and increased mortality [1]. Functionally, the quadriceps is characterised by reduced strength, increased fatigability and decreased endurance, associated with muscle fibre atrophy and a switch towards more glycolytic muscle fibres (types 1-2). The underlying molecular mechanisms of quadriceps dysfunction in COPD are not well clarified.

Myostatin is a member of the transforming growth factor- $\beta$ family, and is a potent negative regulator of muscle mass, as demonstrated in naturally occurring animal and human genetic mutations, and genetic murine models [2]. Myostatin may therefore be a candidate regulator of muscle mass in disorders characterised by muscle atrophy, including COPD.

Few data exist regarding myostatin in COPD, but a recent crosssectional study showed a three-fold increase in vastus lateralis myostatin mRNA transcripts in COPD patients with significant quadriceps weakness compared to healthy controls [3]. However, the relationship between quadriceps myostatin expression and functional characteristics of the muscle is not known. We hypothesised that myostatin expression would negatively correlate with quadriceps strength and exercise capacity in COPD.

18 patients with COPD were enrolled from clinics at the Royal Brompton Hospital (London, UK). Exclusion criteria included exacerbation in the previous 4 weeks, coexisting heart, renal or liver failure, or a systemic inflammatory or metabolic disorder. 16 healthy, age-matched controls were recruited by advertisement. All participants gave written informed consent and the protocol was approved by the local Research Ethics Committee.
The following were measured in study participants: lung function tests; fat-free mass index, calculated from bioelectrical impedance and normalised to height squared; quadriceps muscle

\begin{tabular}{|c|c|c|c|}
\hline & COPD & Controls & p-value \\
\hline Age yrs & $65 \pm 7$ & $65 \pm 7$ & NS \\
\hline Males:females $n$ & $12: 6$ & $9: 7$ & NS \\
\hline BMI $\mathbf{k g} \cdot \mathrm{m}^{-2}$ & $24.5 \pm 4.7$ & $25.7 \pm 4.8$ & NS \\
\hline Fat free mass index & $15.8 \pm 2.3$ & $17.4 \pm 2.5$ & NS \\
\hline $\mathrm{FEV}_{1} \mathrm{~L}$ & $0.93 \pm 0.40$ & $3.03 \pm 0.63$ & $<0.0001$ \\
\hline FEV $_{1} \%$ pred & $35 \pm 15$ & $112 \pm 13$ & $<0.0001$ \\
\hline DL,Co \% pred & $37.9 \pm 14.3$ & $91.3 \pm 16.1$ & $<0.0001$ \\
\hline TLC \% pred & $120 \pm 19$ & $108 \pm 11$ & 0.03 \\
\hline RV \% pred & $193 \pm 51$ & $103 \pm 16$ & $<0.0001$ \\
\hline Quadriceps MVC kg & $29.8 \pm 8.6$ & $37.7 \pm 9.8$ & 0.02 \\
\hline MVC/BMI & $1.2 \pm 0.3$ & $1.5 \pm 0.5$ & 0.04 \\
\hline Quadriceps endurance ${ }^{\#} \mathbf{s}$ & $91 \pm 47$ & $127 \pm 72$ & NS \\
\hline 6MWD m & $362 \pm 145$ & $630 \pm 86$ & $<0.0001$ \\
\hline SGRQ total score & $57 \pm 11$ & $3 \pm 4$ & $<0.0001$ \\
\hline Locomotion time $\min$ & $48 \pm 45$ & $106 \pm 53$ & 0.004 \\
\hline \multicolumn{4}{|c|}{$\begin{array}{l}\text { Data are presented as mean } \pm \mathrm{SD} \text {, unless otherwise stated. COPD: chronic } \\
\text { obstructive pulmonary disease; } \mathrm{BMI} \text { : body mass index; FEV1: forced expiratory } \\
\text { volume in } 1 \mathrm{~s} \text {; \% pred: \% predicted; } D \mathrm{~L}, \mathrm{CO} \text { : diffusing capacity of the lung for } \\
\text { carbon monoxide; TLC: total lung capacity; RV: residual volume; MVC: maximal } \\
\text { voluntary contraction; } 6 \mathrm{MWD} \text { : } 6 \text {-min walk distance; SGRQ: St George's } \\
\text { Respiratory Questionnaire, NS: nonsignificant i.e. } \mathrm{P}>0.05 \text {. \#: defined as the } \\
\text { time for force to fall to } 80 \% \text { of baseline. }\end{array}$} \\
\hline
\end{tabular}

